\title{
UNIQUENESS RESULTS IN NONLINEAR ELLIPTIC PROBLEMS
}

\author{
MASSIMO GROSSI*
}

Abstract. In this paper we condider uniqueness results for the following nonlinear problem

$$
\begin{cases}-\Delta u=f(u) & \text { in } \Omega \\ u>0 & \text { in } \Omega \\ u=0 & \text { on } \partial \Omega\end{cases}
$$

We will show as the geometry of the domain plays a crucial role in this context. We also discuss the nondegeneracy of the solution.

1. Introduction. In this survey we consider uniqueness results for semilinear elliptic problems of the type

$$
\begin{cases}-\Delta u=f(u) & \text { in } \Omega \\ u>0 & \text { in } \Omega \\ u=0 & \text { on } \partial \Omega\end{cases}
$$

where $\Omega$ is a smooth bounded domain of $\mathcal{R}^{N}, N \geq 2$ and $f \in C^{1}(\mathcal{R})$. In this context the shape of the domain and the structure of nonlinearity $f$ plays a crucial role. Indeed, even in the simple case of $f(s)=s^{p}$ we have multiplicity results for domains $\Omega$ with a "rich" topology or suitable geometry (see for example [3] and the references therein). So some restictions on the geometry of the domain is needed.

We point out that an important tool in the uniqueness results seems to be plaied by the linearized equation associated to $(\mathrm{P})$, namely

$$
\begin{cases}-\Delta v=f^{\prime}(u) v & \text { in } \Omega \\ v=0 & \text { on } \partial \Omega\end{cases}
$$

where $u$ is a solution of $(\mathrm{P})$. We say that the solution $u$ of $(P)$ is nondegenerate if $(L)$ admits only the trivial solution $v=0$.

First uniqueness results for $(\mathrm{P})$ were obtained when $\Omega$ is the ball, since in this case it is possible to reduce $(\mathrm{P})$ to an ODE problem via the Gidas-Ni-Nirenberg theorem. Thanks to this reduction some uniqueness results for $(\mathrm{P})$ were deduced for some special nonlinearities. We discuss this in Section 1. In Section 2 we consider the more difficult problem of a nonspherical domain. Of course, the previous approach does not work. At this stage the dimension of the space plays a role. Indeed, the results obtained in literature are weaker if the dimension of the space is greater that two. In this case, $(N \geq 3)$, uniqueness results are obtained in perturbed cases, for example for special nonlinearities $f_{\varepsilon}$ "close" to a suitable one. The reason of this restrictions relies on the difficulty to deduce qualitative properties of the solution of the linearized problem $(\mathrm{L})$.

Finally in Section 4 we consider uniqueness results involving the critical Sobolev exponent.

*Dipartimento di Matematica, Università di Roma "La Sapienza", P.le A.Moro 2, 00185, Roma, Italy (grossi@mat.uniroma1.it). Supported by MURST, Project "Variazional Methods and Nonlinear Differential Equations". 
2. The Radially Symmetric Case. We start this section recalling the following basic results, due to Gidas, Ni and Nirenberg (1979).

THEOREM 2.1. Let us consider the problem $(P)$ where $\Omega$ is convex and symmetric with respect to $x_{i}, 1 \leq i \leq N$. Then $u$ is symmetric with respect to $x_{i}$ and $\frac{\partial u}{\partial x_{i}}<0$ for $x_{i}>0$.

Proof. (see [17]).

Corollary 2.2. Let us consider the problem $(P)$ where $\Omega$ is the ball $|x| \leq R$. Then $u=u(\rho)$ is radial and $u^{\prime}(\rho)<0$ for $\rho>0$.

Proof. (see [17]).

This Corollary is the starting point of the next uniqueness results in a ball. From this, Gidas, Ni and Nirenberg deduced the following result:

THEOREM 2.3. Let us consider the problem $(P)$ with $f(s)=s^{p}, 1<p<\frac{N+2}{N-2}$ and $\Omega$ is the ball $|x| \leq R$. Then there exists only one solution to $(P)$.

Proof. By the previous Corollary we have that $u$ solves the following ordinary differential equation,

$$
\left\{\begin{array}{l}
-u^{\prime \prime}-\frac{N-1}{\rho} u^{\prime}=u^{p} \quad \text { in }|x| \leq R \\
u>0 \text { in }|x| \leq R \\
u^{\prime}(0)=u(R)=0
\end{array}\right.
$$

From the scaling invariance of (2.1) and the analiticity of the solution we get the uniqueness of the solution (see [18] for the details).

Let us denote by $B_{P, r}=\left\{x \in \mathcal{R}^{N}\right.$ such that $\left.|x-P| \leq r\right\}$ and $B_{r}=B_{0, r}$. Moreover let $\lambda_{1}$ be the first eigenvalue of $-\Delta$ in $B_{R}$ with Dirichlet boundary conditions.

In the next years a lot of work was done to obtain uniqueness results for the more general problem

$$
\begin{cases}-\Delta u=u^{p}+\lambda u & \text { in } B_{R} \\ u>0 & \text { in } B_{R} \\ u=0 \text { in } \partial B_{R} & \end{cases}
$$

where $\lambda<\lambda_{1}$ if $1<p<\frac{N+2}{N-2}$ and $0<\lambda<\lambda^{*}$ if $p=\frac{N+2}{N-2}$. Here $\lambda^{*}=\lambda_{1}$ for $N \geq 4$ and $\lambda^{*}=\frac{\lambda_{1}}{4}$ for $N=3$.

Existence results for (2.2) are classical if $1<p<\frac{N+2}{N-2}$. If $p=\frac{N+2}{N-2}$ in [5] it was proved that for $0<\lambda<\lambda^{*}$ there exists at least one solution. Problem (2.2) is not scaling invariant and so it cannot be studied as (2.1). Indeed nontrivial ODE methods are used in order to prove the following results,

Theorem 2.4. Problem (2.2) admits a unique solution for any $\lambda<\lambda_{1}$ if $1<$ $p<\frac{N+2}{N-2}$ and $0<\lambda<\lambda^{*}$ for $p=\frac{N+2}{N-2}$.

A lot of authors give some contribution to this result. We only recall the papers $[28],[1],[37],[34]$. As we remarked, in this papers an important role is played by the nondegeneracity of the solution $u$. 
3. The Nonradial Setting: the case $N=2$.. In this section we will consider the more general case where $\Omega$ has not any radial symmetry. Of course the ODE methods of the previous section are not applicable anymore. On the other hand, Theorem 2.1 continues to have a central role.

First of all we recall the following result

THEOREM 3.1. Let us consider the problem $(P)$, with $N=2, f(s)=s^{p}$ with $p>1$. Here $\Omega$ is convex and symmetric with respect to $x_{i}, 1 \leq i \leq N$. Then $(P)$ admits only one solution.

This result was proved by Dancer in [15] as a consequence of a general theorem contained also in [15] and of the known uniqueness result for the ball.

At this point we would like to quote here that the linearized operator $L$ plays a crucial role in the uniqueness proof of [15]. Some properties of $L$ were studied in [13].

Before proving the main result we need to recall a few facts about the maximum principle for second order elliptic operators of the form $L u=\Delta u+c(x) u$ with $c(x) \in$ $L^{\infty}(D), u \in W_{l o c}^{2, N} \cap C(\bar{D})$.

Definition 3.2. We say that the maximum principle holds for $L$ in $D$ if $L u \leq 0$ in $D$ and $u \geq 0$ on $\partial D$ imply $u \geq 0$ in $D$.

Two well known sufficient conditions for the maximum principle to hold are the following (see [20],[32])

$$
c(x) \leq 0 \text { in } D
$$

(3.2)there exists a function $g \in W_{l o c}^{2, N} \cap C(\bar{D}), g>0$ in $\bar{D}$ such that $L g \leq 0$ in $D$

Now we denote by $\lambda_{1}(L, D)$ the principal eigenvalue of $L$ in $D$. For the meaning and the properties of $\lambda_{1}(L, D)$ we refer to [6], where is also condiderd the case of nonsmooth boundary. In particular we have

Proposition 3.3. The principal eigenvalue $\lambda_{1}(L, D)$ is strictly decreasing in its dependence on $D$ and on the coefficient $c(x)$. Moreover the "refined" maximum principle holds for $L$ in $D$ if and only if $\lambda_{1}(L, D)$ is positive.

We refer to [6] for the definition of "refined" maximum principle which is a generalized formulation of the maximum principle in the case when one cannot prescribe boundary values of the functions involved.

It is important to notice that, by using this generalized definition of the first eigenvalue, it is possible to prove that also the following condition, which is slightly different from (3.2), is sufficient for the maximum principle to hold.

there exists $g \in W_{\text {loc }}^{2, N} \cap C(\bar{D}), g>0$ in $D$ such that $L g \leq 0$ in $D$

We also recall the following sufficient condition for the maximum principle (see [5], [6]) 
Proposition 3.4. There exists $\delta>0$, depending only on $N$, $\operatorname{diam}(D),\|c\|_{L^{\infty}(D)}$ such that the maximum principle holds for $L$ in any domain $D^{\prime} \subset D$ with $\left|D^{\prime}\right|<\delta$.

Finally we remark that regardless of the sign of $c$ if $L u \leq 0$ in $D$ and $u \geq 0$ in $D$ then $u>0$ in $D$ unless $u \equiv 0$ (Strong Maximum Principle).

Now we consider a solution $u \in C^{3}(\Omega) \cap C^{1}(\bar{\Omega})$ of the problem $(P)$. We are interested in studying the linearized problem We have the following theorem, which holds for $N \geq 2$.

THEOREM 3.5. Let $u$ be a solution of $(P)$ with $f(0) \geq 0$ and assume that $\Omega$ is convex in the $x_{1}$ - direction and symmetric with respect to the hyperplane $x_{1}=0$. Then any solution $v$ of $(L)$ is symmetric in $x_{1}$, i.e. $v\left(x_{1}, x_{2}, \ldots, x_{N}\right)=v\left(-x_{1}, x_{2}, \ldots, x_{N}\right)$.

Proof. The proof is the same as the one shown in a lecture of L. Nirenberg in a slightly different case (see also the remark after the proof).

Let us denote a point $x$ in $\mathcal{R}^{N}$ by $\left(x_{1}, y\right), y \in \mathcal{R}^{N-1}$. Applying the symmetry result of Gidas, $\mathrm{Ni}$, Nirenberg to problem $(P)$ we know that $u$ is symmetric with respect to $x_{1}$ and $\frac{\partial u}{\partial x_{1}}>0$ in $\Omega_{1}^{-}=\left\{x=\left(x_{1}, y\right) \in \Omega\right.$ such that $\left.x_{1}<0\right\}$.

We consider the operator

$$
L=\Delta+f^{\prime}(u)
$$

and want to prove that the maximum principle holds for $L$ in $\Omega_{1}^{-}$. To do this we show that the sufficient condition (3.3) is satisfied.

If we set

$$
g=\frac{\partial u}{\partial x_{1}} \text { in } \Omega_{1}^{-}
$$

we have that $g$ satisfies (3.3) since by the Hopf Lemma $\frac{\partial u}{\partial x_{1}} \not \equiv 0$ on $\partial \Omega \cap \partial \Omega_{1}^{-}$(note that we have assumed $f(0) \geq 0$ ). So the maximum principle holds for $L$ in $\Omega_{1}^{-}$.

Now we consider the function

$$
\psi(x)=v\left(x_{1}, y\right)-v\left(-x_{1}, y\right), \quad x=\left(x_{1}, y\right) \in \Omega_{1}^{-}
$$

where $v$ is a solution of $L$. By easy calculation, using that $u$ is symmetric in $x_{1}$, we get

$$
\begin{cases}L \psi=0 & \text { in } \Omega_{1}^{-} \\ \psi=0 & \text { in } \partial \Omega_{1}^{-}\end{cases}
$$

and hence $\psi \equiv 0$ in $\Omega_{1}^{-}$because of the maximum principle. So $v$ is symmetric in $x_{1}$.

REMARK 3.6. Let us consider the following eigenvalue problem

$$
\begin{cases}-\Delta v+v=f^{\prime}(u) v+\mu v & \text { in } \Omega \\ v=0 & \text { on } \partial \Omega\end{cases}
$$

where $u$ is a solution of $P$.

If $\mu<0$ in [2] it is shown thatw is symmetric in $x_{1}$. 
Of course if $\Omega$ is a ball, the previous theorem gives the radial symmetry of $v$. This was already shown by Lin and Ni in [27], using a different argument, for any $\mu \leq 0$.

We make now some important remarks about the nodal set of $v$ that will also be used in the sequel. Let us set

$$
\begin{gathered}
\mathcal{N}=\overline{\{x \in \Omega \text { such that } v(x)=0\}} \\
\widetilde{\Omega}=\{x \in \Omega: v(x) \neq 0\} \\
\Omega_{i}^{-}=\left\{x=\left(x_{1}, \ldots, x_{N}\right) \in \Omega \text { such that } x_{i}<0\right\} \quad i=1, \ldots, N
\end{gathered}
$$

We have

THEOREM 3.7. The following properties hold

i) there cannot exist any component of $\widetilde{\Omega}$ all contained in one $\Omega_{i}^{-}, i=1, \ldots, N$.

ii) if $N=2$ then the origin $(0, \ldots, 0)$ does not belong to $\mathcal{N}$.

iii) if $N=2$ then $\mathcal{N} \cap \partial \Omega=\emptyset$.

Proof. i) Suppose that there exists a component $D$ of $\widetilde{\Omega}$ all contained in $\Omega_{i}^{-}$and $v>0$ in $D$. Then $\lambda_{1}(L, D)=0$ (where $L$ is the operator defined in (3.4)) since $v$ is an eigenfunction of $L$ in $D$ corresponding to the zero eigenvalue and does not change sign in $D$ (being $v=0$ on $\partial \Omega$ we have $v=0$ on $\partial \Omega$ ). On the other hand, in the proof of the previous theorem we have shown that $L$ satisfies the maximum principle in $\Omega_{i}^{-}$ and this implies, by Proposition 3.3, that $\lambda_{1}\left(L, \Omega_{i}^{-}\right)>0$. Then, by monotonicity, also $\lambda_{1}(L, D)$ should be positive which gives a contradiction.

ii) We will show that if $v(0)=0$ then $v \equiv 0$. Suppose $v(0)=0$ and $v \not \equiv 0$ and set $U_{0}=\Omega$. Since $v \not \equiv 0$ and $v(0)=0$ by the Strong Maximum Principle it cannot be $v \leq 0$ in $\Omega$, so that $U_{0}^{+}=\left\{x \in U_{0}: v(x)>0\right\}$ is open and nonempty. Choose a component $A_{1}$ of $U_{0}^{+}$. If $S_{i}, i=1,2$ is the operator that sends a point to the symmetric one with respect to the $x_{1}$-axis, we have that $S_{i}\left(A_{1}\right)$ is also a component of $U_{0}^{+}$because of the symmetry of $v$. It cannot happen that $A_{1} \cap S_{1}\left(A_{1}\right)=\emptyset$ or $A_{1} \cap S_{2}\left(A_{1}\right)=\emptyset$ for otherwise $A_{1}$ or $S_{1}\left(A_{1}\right)$ would be contained in $\Omega_{1}^{-}$, which is impossible by (i). So $A_{1}=S_{1}\left(A_{1}\right)=S_{2}\left(A_{1}\right)$ is symmetric with respect to the coordinate axes and is open and connected, therefore arcwise connected. If we choose four symmetric points $P_{j}, j \in\{1, \ldots, 4\}$ and join them with simple poligonal curves symmetric in pairs, we can costruct a simple closed poligonal curve $C_{1} \subset A_{1}$ which is symmetric with respect to the axes. By the Jordan Curve Theorem $U_{0} \backslash C_{1}$ has two components and, because $C_{1}$ is symmetric, the origin belongs to the component which has not $\partial U_{0}$ as part of the boundary. Let us denote by $U_{1}$ the component that contains 0 and call it the interior of $C_{1}$, while by the exterior of $C_{1}$ we mean the other component. On $\partial U_{1}=C_{1}$ we have $v>0$, so that $v \not \equiv 0$ in $U_{1}$ and, by the Strong Maximum Principle, it is not possible that $v \geq 0$ in $U_{1}$, since $v(0)=0$, so that $U_{1}^{-}=\left\{x \in \Omega_{1}: v(x)<0\right\}$ is open and nonempty. Taking a component $A_{2}$ of $U_{1}^{-}$we observe that $v=0$ on $\partial A_{2}$ because $v \geq 0$ on $\partial U_{1}$ so that $A_{2}$ is also a component of $\widetilde{\Omega}$. As before we can costruct a closed symmetric simple curve $C_{2} \subset A_{2}$ and in the interior $U_{2}$ of $C_{2}$ (the component of $U_{1} \backslash C_{2}$ to which the origin belongs) we can choose a component $A_{3}$ of $U_{2}^{+}=\left\{x \in U_{2} \quad: \quad v(x)>0\right\}$ which is also a component of $\widetilde{\Omega}$. Moreover $A_{3}$ is disjoint from $A_{1}$ because $A_{1}$ contains $C_{1}=\partial \Omega_{1}$ which belongs to the exterior of $C_{2}$. Proceeding in this way we obtain infinitely many disjoint components $\left\{A_{n}\right\}_{n \geq 1}$ of $\widetilde{\Omega}$. 
This is not possible because by Proposition 3.4 there exists $\delta>0$ such that $\left|A_{n}\right| \geq \delta$ for each $n$, otherwise by the Maximum Principle $v$ would be 0 in $A_{n}$, since $v=0$ on $\partial A_{n}$ and $L v=0$ in $A_{n}$ with $L=\Delta-\lambda+f^{\prime}(v)$. Hence there are only finitely many components $A_{n}$ which gives a contradiction.

iii) We will show that in a neighboorhood of $\partial \Omega$ we have $v>0$ or $v<0$. Suppose the contrary and choose a component $A_{1}$ of $U_{0}^{+}=\left\{x \in U_{0}: v(x)>0\right\}$. Since $v=0$ on $\partial \Omega$ we have $v=0$ on $\partial A_{1}$ and as in (ii) we costruct a closed simple curve $C_{1} \subset A_{1}$ symmetric with respect to the axes. In the exterior $U_{1}$ of $C_{1}$, i.e. in the component containing $\partial \Omega$ there are points where $v<0$ by what we assumed. So we can costruct a closed simple curve $C_{2} \subset A_{2}$ where $A_{2}$ is a nonempty component of $U_{1}^{-}=\left\{x \in U_{1} \quad: \quad v(x)<0\right\}$. Proceeding as in the proof of (ii) we obtain infinitely many components of $\widetilde{\Omega}$ which is not possible by Proposition 3.4 , as we remarked before.

REMARK 3.8. If $\Omega$ is a ball in $\mathcal{R}^{N}$, the properties i) - iii) are easy consequences of the radial symmetry of $v$.

Now we consider two solutions $u_{1}$ and $u_{2}$ of the problem $P$ and set

$$
\mathcal{M}=\overline{\left\{x \in \Omega \text { such that } u_{1}(x)=u_{2}(x)\right\}} \quad, \quad \widehat{\Omega}=\left\{x \in \Omega \text { such that } u_{1} \neq u_{2}\right\}
$$

The next theorem contains some information on $\mathcal{M}$ and a uniqueness result.

Theorem 3.9. Suppose that $f$ is convex. Then we have

there cannot exist any component Dof

$\widehat{\Omega}$ all contained in one $\Omega_{i}^{-}, i=1, \ldots, N$.

$$
\begin{gathered}
\text { if } N=2 \text { then } \mathcal{M} \cap \partial \Omega=\emptyset \\
\text { if } N=2 \text { and } \max _{x \in \bar{\Omega}} u_{1}(x)=\max _{x \in \bar{\Omega}} u_{2}(x) \text { then } u_{1} \equiv u_{2}
\end{gathered}
$$

Proof. Set $w(x)=u_{1}(x)-u_{2}(x), x \in \Omega$. Since $f$ is convex $w$ satisfies

$$
\left\{\begin{array}{lr}
\Delta w+f^{\prime}\left(u_{2}\right) w \leq 0 & \text { in } \Omega \\
w=0 & \text { in } \partial \Omega .
\end{array}\right.
$$

and

$$
\left\{\begin{array}{lr}
\Delta w+f^{\prime}\left(u_{1}\right) w \geq 0 & \text { in } \Omega \\
w=0 & \text { in } \partial \Omega .
\end{array}\right.
$$

First we notice that if $w \geq 0$ by (3.10) and the strong maximum principle $w>0$ in $\Omega$ so that $\Omega=\widehat{\Omega}$. Thus we assume that $w$ changes sign in $\Omega$. To prove (3.7) let us argue by contradiction supposing that there exists a component $D$ of $\widehat{\Omega}$ all contained in $\Omega_{i}^{-}$for some $i \in\{1, \ldots, N\}$ and $w>0$ in $D$.

Since in Theorem (3.5) we proved that in $\Omega_{i}^{-}$the maximum principle holds for the operators $L_{i}=\Delta-\lambda+f^{\prime}\left(u_{i}\right) \quad i=1,2$, by Proposition 2.1 we have that $\lambda_{1}\left(L_{1}, \Omega_{i}^{-}\right)>$ 0 , for $i=1,2$. Hence also $\lambda_{1}\left(L_{1}, D\right)>0$ and, again by Proposition (3.3), the "refined" maximum principle holds for $L_{1}$ in $D$. This last fact together with (3.11) would imply 
that $w \leq 0$ in $D$ against what we assumed. If instead we suppose $w \leq 0$ in $D$ then we argue in the same way using the operator $L_{2}$ and (3.10).

To prove (3.8) it is enough to observe that, by the Gidas, Ni and Nirenberg symmetry result, $u_{1}$ and $u_{2}$ are symmetric in any $x_{i}$ and hence so is $w$. Thus arguing as in iii) of the previous theorem the assumption $\mathcal{M} \cap \partial \Omega \neq \emptyset$ would bring a contradiction.

Finally, to prove (3.9), we notice that, again by the Gidas, Ni and Nirenberg result, $\max _{\bar{\Omega}} u_{i}(x)=u_{i}(0), i=1,2$; therefore if the two maxima coincide the origin belongs $x \in \bar{\Omega}$ to $\mathcal{M}$. As in ii) of Theorem 3.7 this gives a contradiction.

Now we prove a generalization of (3.9) of Theorem 3.9.

Let $\Omega$ be as before and $N=2$. Let us call a function $u \in C^{1}(\bar{\Omega})$ symmetric and monotone if $u$ is symmetric in $x_{1}, x_{2}$ and $\frac{\partial u}{\partial x_{i}}>0$ in $\Omega_{i}^{-}, i=1,2$ and let $f: \mathcal{R} \longrightarrow \mathcal{R}$ be a $C^{1}$-function.

THEOREM 3.10. Suppose that $N=2, f$ is convex and $u_{1}, u_{2} \in C^{3}(\Omega) \cap C^{1}(\bar{\Omega})$ are symmetric and monotone functions that satisfy the equation

$$
-\Delta u+\lambda u=f(u) \quad \text { in } \Omega
$$

If $u_{1}(0)=u_{2}(0)$ and $u_{1} \leq u_{2}$ on $\partial \Omega$ then $u_{1}$ and $u_{2}$ coincide.

Proof. As in the proof of Theorem 3.5 we deduce that the operators $L=\Delta-\lambda+$ $f^{\prime}\left(u_{i}\right), i=1,2$ satisfy the maximum principle in $\Omega_{j}^{-}, j=1,2$.

Since the difference $w=u_{1}-u_{2}$ satisfies a linear equation $\Delta w-\lambda w+c(x) w=0$ with $c \in L^{\infty}(\Omega)$ and $f \in C^{1}$ we have that Proposition 3.4 and the strong maximum principle apply to $w$. Arguing as in Theorem 3.7 we first deduce that cannot exist any component $D$ of $\widehat{\Omega}=\left\{x \in \Omega: u_{1} \neq u_{2}\right\}$ such that $u_{1}=u_{2}$ on $\partial D$ and cointained in $\Omega_{j}^{-}, j=1,2$.

Then we can follow exactly the proof of Theorem 3.7 with the only remark that in the first step we choose a component $A_{1}$ of $\Omega_{0}^{+}=\{x \in \Omega: w(x)>0\}$ and we have $w=0$ on $\partial A_{1}$, because of the hypothesis $w(x) \leq 0$ on $\partial \Omega$. So $A_{1}$ is also a component of $\widehat{\Omega}$ with $u_{1}=u_{2}$ on $\partial A_{1}$. The same property holds, by construction, also for the other components $A_{2}, A_{3}$; therefore we conclude as in Theorem 3.7.

REMARK 3.11. If $\Omega$ is a ball then any solution $u$ of $(P)$ is radial and hence the claim of Theorem 3.10 follows immediately from the theory of ordinary differential equation. Therefore this result can be seen as a generalization of the uniqueness theorem for an o.d.e.

Nevertheless it is instructive to see how we can get very easily this result in a ball without using the underlaying ordinary equation but exploiting only maximum principles. Therefore suppose $\Omega=B_{R}(0) \subset \mathcal{R}^{N}$ and $u_{i} \in C^{2}(\bar{\Omega}), i=1,2$, satisfying $-\Delta u=f(u)$ in $\Omega$. Let us prove that if $u_{1}(0)=u_{2}(0)$ then $u_{1} \equiv u_{2}$. In fact the difference $w=u_{1}-u_{2}$ satisfies a linear equation $\Delta w+c(x) w=0$. By Proposition 2.2 there exists $\delta>0$ such that if $0 \leq r_{1}<r_{2}<R$ and $r_{2}-r_{1}<\delta$ then the Maximum Principle holds for $\Delta+c$ in $B_{r_{2}} \backslash B_{r_{1}}$. We claim that $u_{1}$ and $u_{2}$ coincide on $\partial B_{r}$ for any $r<\delta$. In fact it cannot be $u_{1}>u_{2}$ on $\partial B_{r}$ because by Proposition 3.4 and the strong maximum principle it would be $u_{1}>u_{2}$ on $B_{r}$, against the assumption $u_{1}(0)=u_{2}(0)$. In the same way it is not possible that $u_{1}<u_{2}$ on $\partial B_{r}$. So $u_{1} \equiv u_{2}$ in $\bar{B}_{\delta}$. Making the same reasoning in $B_{\frac{3}{2} \delta} \backslash B_{\frac{1}{2} \delta}$ (that has $\partial B_{\delta}$ in the interior) we get $u_{1} \equiv u_{2}$ in $B_{\frac{3}{2} \delta}$ and after a finite number of steps we get $u_{1} \equiv u_{2}$ in $B_{R}$. 
4. The Nonradial Setting: The Case $N \geq 3$. In this section we consider uniqueness problems of $(P)$ for suitable nonspherical domains $\Omega \subset \mathcal{R}^{N}, N \geq 3$. In this context the uniqueness results are weaker than the previous section. Indeed, since the topology of the nodal zones of the solution of $(L)$ with $N \geq 3$ is more complicated than the corresponding two-dimensional case, it seems very difficult to obtain nondegeneracity results for solution of $(P)$. We only know uniqueness results to solution of $(P)$ for perturbed problem, i.e. when the nonlinearity $f=f_{\epsilon}$ and the solution $u_{\epsilon}$ converges to a solution of a limit problem. A first example is the following result, due to E.N. Dancer.

THEOREM 4.1. Let us consider a solution of the following problem

$$
\left\{\begin{array}{lr}
-\varepsilon \Delta u+u=u^{p} & \text { in } \Omega \\
u>0 & \text { in } \Omega \\
u=0 & \text { in } \partial \Omega .
\end{array}\right.
$$

Here $1<p<\frac{N+2}{N-2}$ and $\Omega$ satisfies some geometrical assumptions (see [14]): Then, for $\varepsilon$ small enough, there exists only one solution to (4.1).

Proof. See [14].

Now we come back to the nonlinearity $f(s)=s^{p}$. We recall that, by Pohozaev's identity, in this case there is no solutions to $(P)$ in star-shaped domains for $p \geq \frac{N+2}{N-2}$. Concerning the uniqueness we have the following result (see [22])

THEOREM 4.2. Let us consider the problem

$$
\left\{\begin{array}{lr}
-\Delta u=N(N-2) u^{p} & \text { in } \Omega \\
u>0 & \text { in } \Omega \\
u=0 & \text { in } \partial \Omega .
\end{array}\right.
$$

Let $\Omega \subset \mathcal{R}^{N}, N \geq 3$, be a bounded smooth domain satisfying

$$
\Omega \text { is convex in the } x_{i} \text { direction, } i=1, . ., N \text {. }
$$

and

$$
\text { Sis symmetric with respect to the hyperplanes } x_{i}=0, i=1, . ., N \text {. }
$$

Then there exists $\varepsilon>0$ such that for any $p \in] \frac{N+2}{N-2}-\varepsilon, \frac{N+2}{N-2}[$ there is only one solution to (4.2). Moreover this solution is nondegenerate, i.e. the linear equation

$$
\left\{\begin{array}{lr}
-\Delta v=p N(N-2) u^{p-1} v & \text { in } \Omega \\
v=0 & \text { in } \partial \Omega .
\end{array}\right.
$$

admits only the trivial solution $v \equiv 0$.

In order to prove the previous theorem we need to know with great care the asymptotic behaviour of the solutions of (4.2) as $p \rightarrow \frac{N+2}{N-2}$. We start with the following result

Proposition 4.3. If $\Omega$ satisfies (4.3) and (4.4) then

$$
{\frac{\int_{\Omega}\left|\nabla u_{n}\right|^{2}}{\left(\int_{\Omega}\left|u_{n}\right|^{p_{n}+1}\right)}}^{\frac{2}{p_{n}+1}} \rightarrow S_{N} \text { as } n \rightarrow \infty,
$$


where $S_{N}$ is the best Sobolev constant in $\mathcal{R}^{N}$.

Proof. In the proof of this theorem we use a blow up technique as in the paper of Gidas and Spruck (see [19]) and some important results of [26].

Since $\Omega$ verifies (4.3) and (4.4), using the Pohozaev identity (see [31]) it is not difficult to prove that

$$
u_{n}(0)=\left\|u_{n}\right\|_{\infty} \rightarrow \text { as } n \rightarrow \infty \infty
$$

Let us define

$$
\widetilde{u}_{n}(x)=\frac{1}{\left\|u_{n}\right\|_{\infty}} u_{n}\left(\frac{x}{\left\|u_{n}\right\|_{\infty}^{p_{n}-1}}\right), \quad \widetilde{u}_{n}: \Omega_{n}=\left\|u_{n}\right\|_{\infty}^{\frac{p_{n}-1}{2}} \cdot \Omega \rightarrow \mathcal{R}
$$

By easy calculation $\widetilde{u}_{n}$ satisfies

$$
\left\{\begin{array}{lr}
-\Delta \widetilde{u}_{n}=p N(N-2) \widetilde{u}_{n}^{p_{n}} & \text { in } \Omega_{n} \\
\widetilde{u}_{n}>0 & \text { in } \Omega_{n} \\
\widetilde{u}_{n}=0 & \text { on } \partial \Omega_{n} .
\end{array}\right.
$$

Notice that $\widetilde{u}_{n}(0)=1,0<\widetilde{u}_{n}(x) \leq 1$ for $x \in \Omega_{n}$ and $\Omega_{n}$ converges to $\mathcal{R}^{N}$ (by the notation $\Omega_{n} \rightarrow \mathcal{R}^{N}$ we mean that for any $K \subset \mathcal{R}^{N}$ we have $\Omega_{n} \supset K$ for $n$ large). Again by elliptic theory $\widetilde{u}_{n} \rightarrow U$ in $C^{1}(K)$ for every compact set $K$ of $\mathcal{R}^{N}$, and $U$ solves

$$
\begin{cases}-\Delta U=N(N-2) U^{\frac{N+2}{N-2}} & \text { in } \mathcal{R}^{N} \\ 0 \leq U \leq 1 & \text { in } \mathcal{R}^{N} \\ U(0)=1 & \end{cases}
$$

The solution of (4.10) is unique (see [11]) and

$$
U(x)=\frac{1}{\left(1+|x|^{2}\right)^{\frac{N-2}{2}}}
$$

Now by using some estimates contained in [26] we will prove that

$$
\int_{\Omega}\left|u_{n}\right|^{p_{n}+1} \rightarrow \int_{\mathcal{R}^{N}} U(x)^{\frac{2 N}{N-2}}
$$

First of all we recall the following inequality (see [26]):

$$
u_{n}(x) \leq \frac{C}{\left\|u_{n}\right\|_{\infty}} \frac{1}{|x|^{N-2}} \quad \text { if }|x|<\delta
$$

where $C$ and $\delta$ are positive constant which do not depend on $n$.

From (4.13) we will deduce (4.12). Let us compute

$$
\int_{\Omega}\left|u_{n}\right|^{p_{n}+1}=\int_{|x|<\delta}\left|u_{n}\right|^{p_{n}+1}+\int_{\{|x|>\delta\} \cap \Omega}\left|u_{n}\right|^{p_{n}+1}=I_{n, 1}+I_{n .2}
$$

Let us prove that

$$
I_{n, 1} \rightarrow S_{N}^{N / 2}
$$


and

$$
I_{n, 2} \rightarrow 0
$$

We have

$$
\begin{gathered}
I_{n, 1}=\int_{|x|<\delta}\left|u_{n}(x)\right|^{p_{n}+1}=\left\|u_{n}\right\|_{\infty}^{p_{n}+1-\frac{p_{n}-1}{2} N} \int_{|x|<\delta\left\|u_{n}\right\|_{\infty}^{\frac{p_{n}-1}{2}}}\left|\widetilde{u}_{n}(x)\right|^{p_{n}+1} \\
(4.17)=\left.\left\|u_{n}\right\|_{\infty}^{\frac{N-2}{2}\left(\frac{N+2}{N-2}-p_{n}\right)} \int_{\mathcal{R}^{N}} \widetilde{u}_{n}(x)\right|^{p_{n}+1} \chi_{\left\{|x|<\delta\left\|u_{n}\right\|_{\infty}^{\frac{p_{n}-1}{2}}\right\} .}
\end{gathered}
$$

From (4.13) we get

$$
\tilde{u}_{n}(x) \leq C \frac{1}{\left\|u_{n}\right\|_{\infty}^{2-\frac{p_{n}-1}{2}(N-2)}} \frac{1}{|x|^{N-2}} \leq \frac{C}{|x|^{N-2}} \quad \text { if }|x|<\delta\left\|u_{n}\right\|_{\infty}^{\frac{p_{n}-1}{2}}
$$

Now since $\widetilde{u}_{n}$ is bounded near the origin and $\widetilde{u}_{n} \rightarrow U$ pointwise in $\mathcal{R}^{N}$, from (4.18) and dominate convergence theorem we get

$$
\int_{\mathcal{R}^{N}} \widetilde{u}_{n}(x)^{p_{n}+1} \chi_{\left\{|x|<\delta\left\|u_{n}\right\|_{\infty}^{\frac{p_{n}-1}{2}}\right\}} \rightarrow \int_{\mathcal{R}^{N}} U(x)^{\frac{2 N}{N-2}}
$$

Moreover, again by [26], it is possible to deduce

$$
\left\|u_{n}\right\|_{\infty}^{\frac{N-2}{2}\left(\frac{N+2}{N-2}-p_{n}\right)} \rightarrow \text { as } n \rightarrow \infty
$$

and then

$$
I_{n, 1}=\left\|u_{n}\right\|_{\infty}^{\frac{N-2}{2}\left(\frac{N+2}{N-2}-p_{n}\right)} \int_{\mathcal{R}^{N}} \widetilde{u}_{n}(x)^{p_{n}+1} \chi_{\left\{|x|<\delta\left\|u_{n}\right\|_{\infty}^{\frac{p_{n}-1}{2}}\right\}} \rightarrow S_{N}^{N / 2}
$$

and this proves (4.15). In order to prove (4.16), we remark that from (4.13) it follows

$$
u_{n}(x) \leq \frac{C}{\left\|u_{n}\right\|_{\infty}} \frac{1}{\delta^{N-2}} \quad \text { if }|x|=\delta
$$

From this we deduce that

$$
u_{n}(x) \leq \frac{C}{\left\|u_{n}\right\|_{\infty}} \frac{1}{\delta^{N-2}} \quad \text { in }\{|x|>\} \cap \Omega
$$

Indeed, if by contradiction there exists a point $x_{n} \in\{|x|>\delta\} \cap \Omega$ such that $u_{n}\left(x_{n}\right)>$ $\frac{C}{\left\|u_{n}\right\|_{\infty}} \frac{1}{\delta^{N-2}}$, we would get the existence of a maximum point for $u_{n}$ in $\{|x|>\delta\} \cap \Omega$. But this is not possible by (4.3) and (4.4) and Gidas-Ni-Nirenberg theorem. Hence (4.23) holds and then we get (4.16) and so (4.12).

Finally, since $u_{n}$ is a solution of (4.2) we get

$$
\frac{\int_{\Omega}\left|\nabla u_{n}\right|^{2}}{\left(\int_{\Omega}\left|u_{n}\right|^{p_{n}+1}\right)^{\frac{2}{p_{n}+1}}}=N(N-2)\left(\int_{\Omega}\left|u_{n}\right|^{p_{n}+1}\right)^{1-\frac{2}{p_{n}+1}} \rightarrow S_{N} \text { as } n \rightarrow \infty
$$

and this proves the claim.

Now we recall some results due to Han (see [23], Theorem 1, Lemma 3 and proposition $1)$. 
THEOREM 4.4. Let $\Omega$ be a smooth bounded domain of $\mathcal{R}^{N}$ and $u_{n}$ a solution of (4.2). If $u_{n}$ satisfies

$$
\frac{\int_{\Omega}\left|\nabla u_{n}\right|^{2}}{\left(\int_{\Omega}\left|u_{n}\right|^{p_{n}+1}\right)^{\frac{2}{p_{n}+1}}} \rightarrow S_{N} \text { as } n \rightarrow \infty
$$

where $S_{N}$ is the best Sobolev constant, then (up to a subsequence)

$$
\left(\frac{N+2}{N-2}-p_{n}\right)\left\|u_{n}\right\|_{\infty}^{2} \rightarrow 2 \sigma_{N}^{2}\left[\frac{N(N-2)}{S_{N}}\right]^{N / 2} g\left(x_{0}, x_{0}\right)
$$

where $g(x, y)$ is the regular part of the Green's function $G(x, y)$, i.e.

$$
g(x, y)=G(x, y)-\frac{1}{(N-2) \sigma_{N}|x-y|^{N-2}}
$$

and $\sigma_{N}$ is the area of the unit sphere in $\mathcal{R}^{N}$ and $x_{0}$ is a critical point of $g$. Moreover

$$
u_{n}(x) \leq k \frac{\left\|u_{n}\right\|_{\infty}}{\left(1+\left\|u_{n}\right\|_{\infty}^{\frac{4}{N-2}}\left|x-x_{0}\right|^{2}\right)^{\frac{N-2}{2}}}
$$

and

$$
\left\|u_{n}\right\|_{\infty} u_{n}(x) \rightarrow(N-2) \sigma_{N} G\left(x, x_{0}\right)
$$

in $C^{1}(\omega)$ for any neighborhood $\omega$ of $\partial \Omega$ not containing $x_{0}$.

Finally

(4.30) $\left|\nabla u_{n}\right|^{2} \rightarrow N(N-2)\left[\frac{S_{N}}{N(N-2)}\right]^{N / 2} \delta_{x_{0}} \quad$ in the sense of distributions

REMARK 4.5. If $\Omega$ is a smooth, bounded domain satisfying (4.3) and (4.4) it is not difficult to deduce by Proposition 4.3 that $x_{0}=0$ in Theorem 4.4.

At this stage we are in condition to prove Theorem 4.2.

\section{Proof of Theorem 4.2.}

We argue by contradiction: let us suppose that there exists a sequence $p_{n} \nearrow \frac{N+2}{N-2}$ and functions $u_{n}, v_{n} \in C^{\infty}(\Omega)$ which solve (4.2) with $p$ replaced by $p_{n}$.

Set

$$
\widetilde{w}_{n}(x)=u_{n}\left(\frac{x}{\left\|u_{n}\right\|_{\infty}^{\frac{p_{n}-1}{2}}}\right)-v_{n}\left(\frac{x}{\left\|u_{n}\right\|_{\infty}^{\frac{p_{n}-1}{2}}}\right), \quad \widetilde{w}_{n}: \Omega_{n} \rightarrow \mathcal{R}
$$

As in Theorem 4.2 we have $\left\|u_{n}\right\|_{\infty} \rightarrow \infty$ and $\Omega_{n} \rightarrow \mathcal{R}^{N}$. Moreover

$$
\left\{\begin{array}{lr}
-\Delta \widetilde{w}_{n}=c_{n}(x) \widetilde{w}_{n} & \text { in } \Omega \\
w_{n}=0 & \text { in } \partial \Omega .
\end{array}\right.
$$


where

$$
\begin{array}{r}
c_{n}(x)=N(N-2) p_{n} \int_{0}^{1}\left(\frac{t}{\left\|u_{n}\right\|_{\infty}} u_{n}\left(\frac{x}{\left\|u_{n}\right\|_{\infty}^{\frac{p_{n}-1}{2}}}\right)\right. \\
\left.+\frac{1-t}{\left\|u_{n}\right\|_{\infty}} v_{n}\left(\frac{x}{\left\|u_{n}\right\|_{\infty}^{\frac{p_{n}-1}{2}}}\right)\right)^{p_{n}-1} d t
\end{array}
$$

We have

$$
c_{n}(x) \rightarrow \frac{N(N+2)}{\left(1+|x|^{2}\right)^{2}} \quad \text { uniformly on compact sets of } \mathcal{R}^{N}
$$

So, for $x \in \Omega_{n}$, let us define

$$
w_{n}=\frac{\widetilde{w}_{n}}{\left\|\widetilde{w}_{n}\right\|_{\infty}} \quad, \quad w_{n}: \Omega_{n} \rightarrow \mathcal{R}
$$

Of course $w_{n}$ solves

$$
\left\{\begin{array}{lr}
-\Delta w_{n}=c_{n}(x) w_{n} & \text { in } \Omega \\
\left\|w_{n}\right\|_{\infty}=1 & \text { on } \partial \Omega \\
w_{n}=0 &
\end{array}\right.
$$

and $w_{n}$ is a symmetric function.

Since $w_{n}$ is bounded, using standard elliptic estimates we deduce that $w_{n}$ converges to a symmetric function $w$ uniformly on compact set of $\mathcal{R}^{N}$. Moreover $w$ satisfies

$$
\left\{\begin{array}{l}
-\Delta w=N(N+2) \frac{w}{\left(1+|x|^{2}\right)^{2}} \quad \text { in } \Omega \\
\left\|w_{n}\right\|_{\infty}=1 \\
\|w\|_{\infty} \leq 1
\end{array}\right.
$$

Now we need the following estimate:

$$
\int_{\Omega_{n}}\left|\nabla w_{n}\right|^{2} \leq C
$$

In order to prove (4.38) let us multiply $(4.36)$ by $w_{n}$. Then by Sobolev inequality and for some $0<\delta<\frac{4}{N-2}$

$$
S_{N}\left(\int_{\Omega_{n}}\left|w_{n}\right|^{2^{*}}\right)^{\frac{2}{2^{*}}} \leq \int_{\Omega_{n}}\left|\nabla w_{n}\right|^{2}=\int_{\Omega_{n}}\left|c_{n}(x)\right| w_{n}^{2} \leq \int_{\Omega_{n}}\left|c_{n}(x)\right| w_{n}^{2-\delta}
$$

since $\left\|w_{n}\right\|_{\infty}=1$. Then using Hölder inequality and (4.32)

$$
\begin{aligned}
& S_{N}\left(\int_{\Omega_{n}}\left|w_{n}\right|^{2^{*}}\right)^{\frac{2}{2^{*}}} \leq\left(\int_{\Omega_{n}}\left|w_{n}\right|^{2^{*}}\right)^{\frac{2-\delta}{2^{*}}} \leq\left(\int_{\Omega_{n}} c_{n}(x)^{\frac{2^{*}}{2^{*}-2+\delta}}\right)^{\frac{2^{*}-2+\delta}{2^{*}}} \leq \\
& \leq C\left(\int_{\Omega_{n}}\left|w_{n}\right|^{2^{*}}\right)^{\frac{2-\delta}{2^{*}}}\left(\int_{\Omega_{n}} \frac{1}{\left(1+|x|^{2}\right)^{\left(p_{n}-1\right) \frac{N-2}{2} \frac{2^{*}}{2^{*}-2+\delta}}}\right)^{\frac{2^{*}-2+\delta}{2^{*}}}
\end{aligned}
$$


and then

$$
\begin{aligned}
& C\left(\int_{\Omega_{n}}\left|w_{n}\right|^{2^{*}}\right)^{\frac{\delta}{2^{*}}} \leq\left(\int_{\Omega_{n}} \frac{1}{\left(1+|x|^{2}\right)^{\left(p_{n}-1\right) \frac{N-2}{2} \frac{2^{*}}{2^{*}-2+\delta}}}\right)^{\frac{2^{*}-2+\delta}{2^{*}}} \leq \\
& \leq\left(\int_{\mathcal{R}^{N}} \frac{1}{\left(1+|x|^{2}\right)^{\left(p_{n}-1\right) \frac{N-2}{2} \frac{2^{*}}{2^{*}-2+\delta}}}\right)^{\frac{2^{*}-2+\delta}{2^{*}}}<\infty
\end{aligned}
$$

since $0<\delta<\frac{4}{N-2}$. So $\int_{\Omega_{n}}\left|w_{n}\right|^{2^{*}}$ is bounded and then again by (4.36) the claim follows.

The structure of the solutions of (4.37) satisfying ((4.38)) was described in [4], where was proved that the following cases occur:

i) $w=0$,

ii) $w=\frac{\partial U}{\partial x_{i}}, \quad i=1, . ., N$ where $U(x)=\left(\frac{1}{1+|x|^{2}}\right)^{\frac{N-2}{2}}$.

or

iii) $w=\frac{1-|x|^{2}}{\left(1+|x|^{2}\right)^{N / 2}}$

So we have that $w(x)=\sum_{i=1}^{N} \alpha_{i} \frac{x_{i}}{\left(1+|x|^{2}\right)^{N / 2}}+\beta \frac{1-|x|^{2}}{\left(1+|x|^{2}\right)^{N / 2}}$. It is easily seen that the study of $w$ is equivalent to consider i), ii), iii) separately.

Now we will prove that in any case a contradiction arises.

Case i) $w=0$

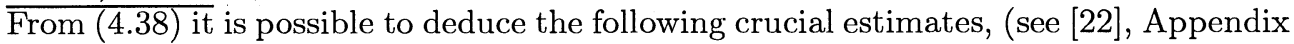
2):

$$
\left|w_{n}(x)\right| \leq \frac{C}{\left(1+|x|^{2}\right)^{\frac{N-2}{2}}} \quad \text { for any } x \in \mathcal{R}^{N}
$$

From (4.42) a contradiction follows easily. Indeed since $\left\|w_{n}\right\|_{\infty}=1$ we can assume that $\exists x_{n} \in \Omega_{n}$ such that $\max _{x \in \Omega_{n}} w_{n}(x)=w_{n}\left(x_{n}\right)=1$ and $\left\|x_{n}\right\|_{\mathcal{R}^{N}} \rightarrow \infty$ because $w_{n} \rightarrow 0$ uniformly on any compact set of $\mathcal{R}^{N}$. But this is not possible by (4.42).

Case ii) $w=\frac{\partial U}{\partial x_{i}}, \quad i=1, . ., N$

In this case we have a contradiction since $w=\frac{\partial U}{\partial x_{i}}$ is not a symmetric function, i.e. does not satisfy $w\left(x_{1}, \ldots,-x_{i-1}, x_{i}, x_{i+1}, \ldots, x_{N}\right)=w(x)$ for any $x \in \mathcal{R}^{N}$.

Case iii) $w=\frac{1-|x|^{2}}{\left(1+|x|^{2}\right)^{N / 2}}$

In what follows we will use Theorem 4.4, where the point $x_{0}=O$ (see Remark 4.5). First of all we notice that, for any neighborhood $\varnothing$ of $\partial \Omega$ not containing $\mathrm{O}$ it holds that

$$
\left\|u_{n}\right\|_{\infty}^{2} \frac{u_{n}(x)-v_{n}(x)}{\left\|u_{n}-v_{n}\right\|_{\infty}} \rightarrow-(N+2) \sigma_{N} G(x, 0) \text { in } C^{1}(\varnothing)
$$

where, as in Theorem $4.4, G$ is the Green function.

Let us prove (4.2). We have

$$
-\Delta\left(\left\|u_{n}\right\|_{\infty}^{2} \frac{u_{n}(x)-v_{n}(x)}{\left\|u_{n}-v_{n}\right\|_{\infty}}\right)=\left\|u_{n}\right\|_{\infty}^{2} d_{n}(x) \frac{u_{n}(x)-v_{n}(x)}{\left\|u_{n}-v_{n}\right\|_{\infty}}
$$

where $d_{n}(x)=N(N-2) p_{n} \int_{0}^{1}\left(t u_{n}(x)+(1-t) v_{n}(x)\right)^{p_{n}-1} d t$. 
So, using (4.28), (4.42) and the dominate convergence theorem we obtain

$$
\begin{gathered}
\left\|u_{n}\right\|_{\infty}^{2} \int_{\Omega} d_{n}(x) \frac{u_{n}(x)-v_{n}(x)}{\left\|u_{n}-v_{n}\right\|_{\infty}}=\left\|u_{n}\right\|_{\infty}^{2-\frac{p_{n}-1}{2} N} \int_{\Omega_{n}} d_{n}\left(\frac{x}{\left\|u_{n}\right\|_{\infty}^{\frac{p_{n}-1}{2}}}\right) w_{n}(x)= \\
(4.45)=\left\|u_{n}\right\|_{\infty}^{p_{n}+1-\frac{p_{n-1}}{2} N} \int_{\Omega_{n}} c_{n}(x) w_{n}(x) \rightarrow-(N+2) \sigma_{N}
\end{gathered}
$$

(the last integral can easily be computed by recalling that $-\Delta w=\frac{1}{\left(1+|x|^{2}\right)^{2}} w$ ).

Moreover, again by (4.28), (4.42) and for any $x \neq 0$

$$
\begin{aligned}
& \left\|u_{n}\right\|_{\infty}^{2} d_{n}(x) \frac{u_{n}(x)-v_{n}(x)}{\left\|u_{n}-v_{n}\right\|_{\infty}} \\
& \leq C \frac{\left\|u_{n}\right\|_{\infty}^{2}}{\left\|u_{n}\right\|_{\infty}^{\left(p_{n}-1\right)\left(\frac{N-2}{2}\left(p_{n}-1\right)-1\right)}} \frac{w_{n}\left(\left\|u_{n}\right\|_{\infty}^{\frac{p_{n}-1}{2}} x\right)}{|x|^{(N-2)\left(p_{n}-1\right)}} \leq \\
& \leq \frac{C}{\left\|u_{n}\right\|_{\infty}^{\left(p_{n}-1\right)\left(\frac{N-2}{2} p_{n}-1\right)-2}} \frac{1}{|x|^{(N-2) p_{n}}} \leq \frac{C}{|x|^{(N-2) p_{n}}}
\end{aligned}
$$

Finally since, in the sense of distributions

$$
\left\|u_{n}\right\|_{\infty}^{2} \int_{\Omega} d_{n}(x) \frac{u_{n}(x)-v_{n}(x)}{\left\|u_{n}-v_{n}\right\|_{\infty}} \phi(x) \rightarrow-(N+2) \sigma_{N} \delta_{0}
$$

by Lemma 2 of [23] we get (4.43).

Now let us write down the Pohozaev identity for $u_{n}$ and $v_{n}$ (see [31])

$$
\begin{aligned}
& \frac{1}{2} \int_{\partial \Omega}(x \cdot \nu)\left(\frac{\partial u_{n}}{\partial \nu}\right)^{2}=\left(\frac{N}{p_{n}+1}-\frac{N-2}{2}\right) \int_{\Omega} u_{n}^{p_{n}+1} \\
& \frac{1}{2} \int_{\partial \Omega}(x \cdot \nu)\left(\frac{\partial v_{n}}{\partial \nu}\right)^{2}=\left(\frac{N}{p_{n}+1}-\frac{N-2}{2}\right) \int_{\Omega} v_{n}^{p_{n}+1}
\end{aligned}
$$

By (4.48) and (4.49) we obtain

$$
\begin{aligned}
& \frac{1}{2} \int_{\partial \Omega}(x \cdot \nu) \frac{\partial}{\partial \nu}\left(\left\|u_{n}\right\|_{\infty}^{2} \frac{u_{n}(x)-v_{n}(x)}{\left\|u_{n}-v_{n}\right\|_{\infty}}\right) \frac{\partial}{\partial \nu}\left[\left\|u_{n}\right\|_{\infty}\left(u_{n}+v_{n}\right)\right]= \\
& =\frac{\frac{N+2}{N-2}-p_{n}}{2(N-2)\left(p_{n}+1\right)}\left\|u_{n}\right\|_{\infty}^{2} \int_{\Omega} h_{n}(x)\left\|u_{n}\right\|_{\infty} \frac{u_{n}(x)-v_{n}(x)}{\left\|u_{n}-v_{n}\right\|_{\infty}}
\end{aligned}
$$

with $h_{n}(x)=\left(p_{n}+1\right) \int_{0}^{1}\left(t u_{n}(x)+(1-t) v_{n}(x)\right)^{p_{n}} d x$. Then from (4.26), (4.29) and (4.42) we get

$$
C \int_{\partial \Omega}(x \cdot \nu)\left(\frac{\partial G(x, 0)}{\partial \nu}\right)^{2}+o(1)=C \int_{\Omega} h_{n}(x)\left\|u_{n}\right\|_{\infty} \frac{u_{n}(x)-v_{n}(x)}{\left\|u_{n}-v_{n}\right\|_{\infty}}+o(1)
$$




$$
\begin{aligned}
= & \frac{C}{\left\|u_{n}\right\|_{\infty^{\frac{p_{n}-1}{2}} N-\left(p_{n}+1\right)}} \int_{p_{p_{n}-1}} \int_{\Omega}^{1}\left(\frac{t}{\left\|u_{n}\right\|_{\infty}} u_{n}\left(\frac{x}{\left\|u_{n}\right\|_{\infty}^{\frac{p_{n}-1}{2}}}\right)\right. \\
& \left.+\frac{(1-t)}{\left\|u_{n}\right\|_{\infty}} v_{n}\left(\frac{x}{\left\|u_{n}\right\|_{\infty^{2}}^{\frac{p_{n}-1}{2}}}\right)\right) w_{n}(x)+o(1) \\
= & C \int_{\mathcal{R}^{N}} \frac{1-|x|^{2}}{\left(1+|x|^{2}\right)^{N+1}}+o(1)=C \int_{0}^{1} \frac{1-\rho^{2}}{\left(1+\rho^{2}\right)^{N+1}} \rho^{N-1} \\
& +C \int_{1}^{\infty} \frac{1-\rho^{2}}{\left(1+\rho^{2}\right)^{N+1}} \rho^{N-1}+o(1)=C \int_{1}^{\infty} \frac{1-\frac{1}{\rho^{2}}}{\left(1+\frac{1}{\rho^{2}}\right)^{N+1}} \frac{1}{\rho^{N+1}} \\
& +C \int_{1}^{\infty} \frac{1-\rho^{2}}{\left(1+\rho^{2}\right)^{N+1}} \rho^{N-1}+o(1)=o(1)
\end{aligned}
$$

again by the dominate convergence theorem. Finally, since (see [8] or [23])

$$
\int_{\partial \Omega}(x \cdot \nu)\left(\frac{\partial G(x, 0)}{\partial \nu}\right)^{2}=(2-N) g(0,0)>0
$$

we have a contradiction. This proves iii) and hence the uniqueness result .

5. The Nonradial Setting: The Critical Case.. In this section we consider uniqueness and nondegeneracy results for a classical elliptic equation involving critical Sobolev exponent, namely

$$
\left\{\begin{array}{lc}
-\Delta u=N(N-2) u^{p}+\varepsilon u & \text { in } \Omega \\
u>0 & \text { in } \Omega \\
u=0 & \text { on } \partial \Omega
\end{array}\right.
$$

where $p=\frac{N+2}{N-2}$. It is well known by the theorem of Brezis and Nirenberg (see [7]) that if $N \geq 4$ and for $0<\varepsilon<\lambda_{1}$, there exists a solution of (5.1) while, if $\varepsilon=0$ and the domain is starshaped, the Pohozaev identity shows that there is not any solution. The asymptotic behaviour of the solution $u_{\varepsilon}$ was studied in [33] where it was proved that, for $\varepsilon \rightarrow 0, u_{\varepsilon}$ concentrates around a critical point of the Robin function. We recall that, if $g(x, y)$ is the regular part of the Greeen function for the laplacian with zero boundary condition, then the Robin function $\psi(x)$ is defined by

$$
\psi(x)=g(x, x)
$$

Converesely, in [21] it is shown that if $N \geq 5$, for any nondegenerate critical point of the function $\psi(x)$ there is only one solution $u_{\varepsilon}$ of (5.1) with the property that $u_{\varepsilon}$ concentrates at $x_{0}$. In the next theorem we state a more general uniqueness result

THEOREM 5.1. Let $\Omega$ be a smooth and bounded domain of $\mathcal{R}^{N}$ with $N \geq 5$, such that it is symmetric with respect to the coordinate hyperplanes and convex in the $x_{k}-$ directions. Let us suppose that $u_{\varepsilon}$ and $v_{\varepsilon}$ are two solutions of (5.1). Then, there exists $\varepsilon_{0}>0$ such that for $\varepsilon<\varepsilon_{0}$,

$$
u_{\varepsilon}=v_{\varepsilon}
$$


Moreover this solution is nondegenerate.

Proof. In [9] this result was firstly proved by assuming that

$$
\lim _{\varepsilon \rightarrow 0} \frac{\int_{\Omega}\left|\nabla u_{\varepsilon}\right|^{2} d x}{\left(\int_{\Omega}\left|u_{\varepsilon}\right|^{p+1}\right)^{2 / p+1} d x}=S_{N}
$$

Assumption (5.4) was removed in [12]. The proof of this results follow the lines of the theorem of the previous section and so we omit it. We only show the nondegeneracity. First of all... Let us denote by $\lambda_{2, n}$ the second eigenvalue of the operator $\Delta+N(N-$ 2) $p_{n} u_{n}^{p_{n}-1}$. Then

$$
\lambda_{2, n}>0
$$

Proof. Since the solution of (2.1) is unique for $n$ large, we can obtain it as a mountain pass solution of the following functional

$$
F(u)=\int_{\Omega}\left|\nabla u_{n}\right|^{2}-\frac{1}{p_{n}+1} \int_{\Omega}\left(u_{n}^{+}\right)^{p_{n}+1}
$$

Then, by a Hofer's result (see [24]), we get that $\lambda_{2, n} \geq 0$.

Now we suppose, by contradiction, that $\lambda_{2, n}=0$. Again let us set

$$
\widetilde{v}_{2, n}(x)=\frac{1}{\left\|u_{n}\right\|_{\infty}} v_{2, n}\left(\frac{x}{\left\|u_{n}\right\|_{\infty}^{p_{n}-1}}\right) \quad, \quad \widetilde{v}_{2, n}: \Omega_{n} \rightarrow \mathcal{R}
$$

and

$$
w_{2, n}=\frac{\widetilde{v}_{2, n}}{\left\|\widetilde{v}_{2, n}\right\|_{\infty}}
$$

We get that $w_{2, n}$ satisfies

$$
\left\{\begin{array}{lr}
-\Delta w_{2, n}=N(N-2) p_{n} \widetilde{u}_{n}^{p_{n}-1} w_{2, n} & \text { in } \Omega_{n} \\
w_{2, n}=0 & \text { on } \partial \Omega .
\end{array}\right.
$$

So $w_{n}$ converges to function $w$ uniformly on compact set of $\mathcal{R}^{N}$, where

i) $w=0$,

ii) $w=\frac{\partial U}{\partial x_{i}}, \quad i=1, . ., N$ where $U(x)=\left(\frac{1}{1+|x|^{2}}\right)^{\frac{N-2}{2}}$,

or

iii) $w=\frac{1-|x|^{2}}{\left(1+|x|^{2}\right)^{N / 2}}$

As in the previous section the study of $w$ can be reduced to the study of i), ii), iii).

Case i) is treated analogously to case i) of Section 3, i.e. using the estimate

$$
\left|w_{n}(x)\right| \leq \frac{C}{|x|^{N-2}} \quad \text { for } x \in \Omega_{n} \cap\{|x|>1\}
$$

whose proof is the same as that (4.42).

Case ii) cannot occur because of Theorem (3.5).

In order to avoid case iii) we follow an idea of Zhang (see [38]). Since $v_{2, n}$ is the second eigenfunction of the operator $\Delta+N(N-2) p_{n} u_{n}^{p_{n}-1}$ then it has two nodal zones. 
Now we remark that, since $w_{2, n} \rightarrow \frac{1-|x|^{2}}{\left(1+|x|^{2}\right)^{N / 2}}$ uniformly on $B(0,2)$ we get for $n$ large and $\imath^{1}$

$$
\left\{\begin{array}{lr}
w_{2, n}(x)>0 & \text { on }|x|=\frac{\delta}{2} \\
w_{n}(x)<0 & \text { on }|x|=\frac{3 \delta}{2}
\end{array}\right.
$$

This implies that

$$
\begin{cases}v_{2, n}>0 & \text { on }|x|=\frac{\delta}{2\left\|u_{n}\right\|_{\infty}^{p_{n}-1}} \\ v_{2, n}<0 & \text { on }|x|=\frac{3 \delta}{2\left\|u_{n}\right\|_{\infty}^{\frac{p_{n}-1}{2}}}\end{cases}
$$

Hence, since $v_{2, n}$ has two nodal zone, $\frac{\partial v_{2, n}}{\partial \nu}$ does not change sign on the boundary of $\Omega$. Finally a contradiction follows by the identity

$$
\int_{\partial \Omega}(x \cdot \nu) \frac{\partial v_{2, n}}{\partial \nu} \frac{\partial u_{n}}{\partial \nu}=0
$$

which can be obtained by considering the function $\eta=x \cdot \nabla u_{n}$ which satisfies

$$
-\Delta \eta=N(N-2) p_{n} u_{n}^{p_{n}-1} \eta+2 u_{n}^{p_{n}}
$$

Then multiplying (4.1) by $\eta$ and (4.30) by $v_{2, n}$ we obtain (5.13).

\section{REFERENCES}

[1] Adimurthi, YadaVA S.L., An elementary proof of the uniqueness of positive radial solutions of a quasilinear Dirichlet problem, Arch. Rat. Mech. Anal., 126 (1994), pp. 219-229.

[2] BABIN A., Symmetry of instability for scalar equations in symmetric domains, J. Diff. Eqns, 123 (1995), pp. 122-152.

[3] Benci V., Cerami G, The effect of the domain topology on the number of positive solutions of nonlinear elliptic problems, Arch. Rational Mech. Anal., 114 (1991), pp. 79-93.

[4] Bianchi G., Egnell H., A note on the sobolev inequality, J. Funct. Anal, 100 (1991), pp. 18-24.

[5] Berestycki H. ,Nirenberg L., On the method of moving planes and the sliding method, Bol. Soc. Bras. Mat., 22 (1991), pp. 1-37.

[6] Berestycki H., Nirenberg L., S.N.S. VARAdhan, The principle eigenvalues and maximum principle for second order elloptic operators in general domains, Comm. Pure. Appl. Math., 47 (1994), pp. 47-92.

[7] Brezis H., Nirenberg L., Positive solutions of nonlinear elliptic equations involving critical Sobolev exponent, Comm. Pure. Appl. Math., 36 (1983), pp. 437-477.

[8] Brezis H., Peletier L., Asymptotics for elliptic equations involving critical growth, Partial Differential Equations and Calculus of Variations, vol I, Birkhauser, Boston, (1989), pp. 149-192.

[9] Cerqueti K., A uniqueness result for a semilinear elliptic equation involving the critical Sobolev exponent in symmetric domains, Asymptotic Analysis, to appear.

[10] CAO D., Noussair E., YAN S., Existence and uniqueness results on single-peaked solutions of a semilinear problem, Ann. Inst. H. Poincaré, 15 (1998), pp. 73-111.

[11] Caffarelli L., Gidas B., Spruck J., Asymptotic symmetry amd local behaviour of semilinear elliptic equations with critical growth, Comm. Pure. Appl. Math., 42 (1989), pp. 271-297.

[12] Cerqueti K., Grossi M., Local estimates for a semilinear elliptic equation with Sobolev critical exponent and applications to a uniqueness result, Nonlinear Diff. Eqns. Appl., to appear.

[13] Damascelli L., Grossi M., Pacella F., Qualitative properties of positive solutions of elliptic equations in symmetric domains via the maximum principle, Ann. Ist. H. Poincaré, 16 (1999), pp. 631-652.

[14] DANCER E.N., On the uniqueness of the positive solution of a singularly perturbed problem, Rocky Mountain J. Math, 25 (1995), pp. 957-975. 
[15] DANCER E.N., The effect of domain shape on the number of positive solutions of certain nonlinear equations, J. Diff. Eqns, 74 (1988), pp. 120-156.

[16] DiNG W.Y., Ni W.M., On the existence of positive entire solutions of a semilinear elliptic equation, Arch. Rat. Mech. Anal., 91 (1986), pp. 283-308.

[17] GidAs B., Ni W. N., NiREnBerg L. , Symmetry and related properties via the maximum principle, Comm. Math. Phys., 68 (1979), pp. 209-243.

[18] Gidas B., Ni W.M., Nirenberg L., Symmetry of positive solutions of nonlinear elliptic equations in $\mathcal{R}^{N}$, Mathematical analysis and applications, Part A, Adv. Math. Suppl. Studies, $7 \mathrm{~A}$, Acad. Press, New York, 1981.

[19] Gidas B., SpRuck J., A priori bounds for positive solutions of nonlinear elliptic equations, Comm. Part. Diff. Eqns.,6 (1981), pp. 883-901.

[20] Gilbarg D., Trudinger N., Elliptic partial differential equations of second order, Berlin Heidelberg New. York, Springer, 1977.

[21] Glangetas L., Uniqueness of positive solutions of a nonlinear elliptic equation involving the critical exponent, Nonlinear Anal., 20 (1993), pp. 571-603.

[22] Grossi M., A uniqueness result for a semilinear elliptic equation in symmetric domains, Adv. Diff. Eqns., 5 (2000), no. 1-3, pp. 193-212.

[23] HAN Z.C., Asymptotic approach to singular solutions for nonlinear elliptic equations involving critical Sobolev exponent, Ann. Ist. H. Poincaré, 8 (1991), pp. 159-174.

[24] Hofer H., A note on the topological degree at a critical point of mountainpass-type, Proc. Amer. Mat. Soc., 90 (1984), pp. 309-315.

[25] Kwong M.K., Uniqueness of positive solutions of $\Delta u-u+u^{p}=0$ in $\mathcal{R}^{n}$, Arch. Rat. Mech. Anal., 105 (1989), pp. 243-266.

[26] Li Y.Y., Prescribing scalar curvature on $S^{N}$ and related problems, Part I, J. Diff. Eqns., 120 (1995), pp. 319-410.

[27] C.S. LIN, W.M. NI, A counterexample to the nodal domain cinjecture and a related semilinear equation, Proc. Amer. Math. Soc., 102 (1988), pp. 271-277.

[28] Ni W.M., Nussbaum R.D., Uniqueness and nonuniqueness for positive radial solutions of $-\Delta u+f(r, u)=0$, Comm. Pure Math. Appl., 38 (1985), pp. 67-108.

[29] Ni W.M., TAKAGI I., On the shape of least energy solutions to a semilinear Neumann problem, Comm. Pure Math. Appl., 41 (1991), pp. 819-851.

[30] NI W.M., WEI J., On the location and profile of spike-layer solutions to singularly perturbed semilinear Dirichlet problems, Comm. Pure Math. Appl., 48 (1995), pp. 731-768.

[31] Pohozaev S., Eigenfunction of the equation $\Delta u+f(u)=0$, Soviet. Math. Dokl., 6 (1965), pp. 1408-1411.

[32] Protter M.H., Weinberger H.F., Maximum principle in differential equations, Prentice Hall, Englewood Cliffs, New Jersey, (1967).

[33] REY O., The role of the Green's function in a nonlinear elliptic equation involving the critical sobolev exponent, J. Funct. Anal., 89 (1990), pp. 1-52.

[34] SRIKANth P.N., Uniqueness of solutions of nonlinear Dirichlet problems, Diff. and Int. Eqs., 6 (1993), pp. 663-670.

[35] J. WEI , On the interior spike layer solutions of singularly perturbed semilinear Neumann problem, Tohoku Math. J., 50 (1998), pp. 159-178.

[36] J. WEI , On single interior spike solutions of Gierer-Meinhardt system:uniqueness, spectrum estimates and stability analysis, Euro. Jour. of Appl. Math., 109 (1999), pp. 353-378.

[37] ZhANG L., Uniqueness of positive solutions of $\Delta u+u+u^{p}=0$ in a ball, Comm. Part. Diff. Eqs, 17 (1992), pp. 1141-1164.

[38] Zhang L., Uniqueness of positive solutions of $\Delta u+u^{p}=0$ in a convex domain in $\mathcal{R}^{2}$, Preprint. 